\title{
Dopage: rien de changé depuis Rome et «panem et circenses»?
}

Jean Martin

Correspondance:

Dr Jean Martin

La Ruelle 6

CH-1026 Echandens

jean.martin@urbanet.ch
De diverses façons, le dopage a une nouvelle fois été à l'ordre du jour cet été, entre autres avec les tests positifs de Floyd Landis, vainqueur du Tour de France 2006, et les doutes autour de l'athlète Marion Jones. A ce sujet: "comment ne pas s'inquiéter de l'absence quasi générale du discours et de la décision politiques. Tout se passe comme si les jeux du cirque, quelle que soit l'évolution de leur règles, confortaient le pouvoir politique», écrit Jean-Yves Nau, chroniqueur français connu, dans la Revue médicale suisse du 23 août 2006. Au même moment, dans Le Temps (Genève) du 25 août, Yves Guisan, membre du Comité central de la FMH et conseiller national, critique l'état des lieux chez nous, stigmatisant la mollesse des milieux sportifs comme des autorités, relevant les contraintes que représentent des données juridiques comme le secret médical. On se souvient d'un cycliste du Tour de Romandie que les médecins du CHUV à Lausanne, ont sauvé de justesse il y a une dizaine d'années; concerné professionnellement à l'époque, j'avais été frappé par la tension entre l'intérêt public à lutter contre le dopage et l'exigence de respect de la sphère privée.

La question est difficile: on a le droit de se mettre en danger en faisant de l'escalade, de la course motorisée ou simplement en fumant. Alors, si beaucoup d'athlètes se dopent ou se laissent doper, qui nous donne le droit de vouloir préserver leur santé contre leur gré? On entend même tel confrère dire qu'il y avait dopage depuis l'Antiquité et qu'il convient de permettre ces pratiques, sous «garantie» (!) de supervision médicale. Mais comment ne pas voir que le dopage contrôlé, "propre en ordre», demanderait cependant comme aujourd'hui d'établir des limites (listes de produits, dosages, fréquences ...) - pour le moins à l'usage des médecins. L'opposition de principe au dopage, bien sûr, découle aussi d'une volonté d'égalité des chances des sportifs qui s'alignent dans une épreuve. Et je refuse l'argument selon lequel on devrait autoriser celui qui est moins bien servi par la nature à «combler son retard» par des moyens artificiels.

Arrêtons-nous à l'interpellation de J.-Y. Nau mise en exergue. Souvent, on décrit la lutte contre le dopage comme une partie de gen- darmes et voleurs, les gendarmes ayant toujours une ou plusieurs longueurs de retard sur les coachs et athlètes et sur les pharmaco-chimistes qui les aident. Il y a de cela sans doute mais les manques sont-ils seulement ou surtout techniques? Est-il contestable que, aujourd'hui comme

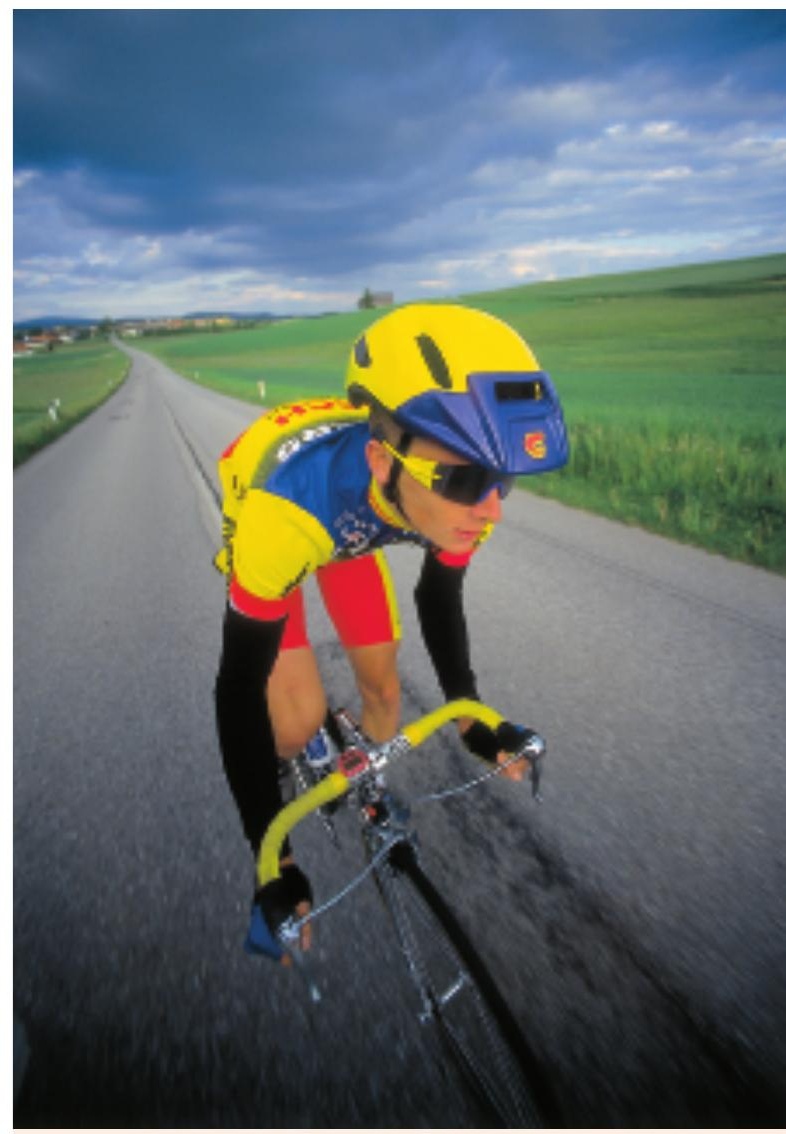

Si beaucoup d'athlètes se dopent ou se laissent doper, qui nous donne le droit de vouloir préserver leur santé contre leur gré?

à Rome, une population nourrie de pain et de jeux se laisse plus facilement mener, voire endormir? Certaines récupérations politiques des exploits d'équipes nationales au Mondial de football récent ont été spectaculaires (randonnant cet été au Grand-St-Bernard, j'ai vu que les drapeaux officiels de la douane italienne étaient nouveaux et portaient - en plus - l'image du trophée obtenu par la squadra!). A fin septembre, un journaliste commentant la prochaine élection 
présidentielle au Brésil estimait que la passion pour le sport, football en particulier, était un facteur expliquant une certaine apathie devant les résultats bien insatisfaisants de la gestion gouvernementale. Il faut voir aussi que le pays qui luttera particulièrement efficacement contre le dopage verra diminuer ses chances de médailles lors des compétitions internationales. Et il n'y pas que le politique qui aime les médailles: le public s'émeut de ce que le dopage est répandu mais nous y contribuons tous quand, devant la télévision ou au bord des routes des Tours, nous demandons des performances, toujours plus vite, toujours plus loin.

Autre son de cloche sur le manque d'efficacité des pouvoirs publics devant les défis de l'époque: «L'immobilisme nous berne», dit Erhard Taverna dans le Bulletin des médecins suisses, aussi le 23 août; «Tant que nous répartirons les risques globaux en petits paquets faciles à manier, la somme des efforts n'aura pas assez d'effet. Faire plus contreviendrait à la règle universelle: business as usual» (on continue comme si de rien n'était). Dans ce sens, le dopage serait un petit paquet, quoique pas facile à manier! C'est là un autre aspect des enjeux: trouver un équilibre adéquat entre des efforts prioritaires sur tel créneau comme le dopage, et une action de fond pour changer les règles du jeu au sens le plus large, pour aller vers des pratiques moins dures, plus conviviales, dans le sport comme d'autres secteurs de la vie collective. Les médecins ont ici évidemment un rôle à jouer.

PS: Au moment où ce texte va sous presse, les journaux de 23 septembre 2006 nous apprennent que le Conseil fédéral a décidé de dynamiser la lutte antidopage et qu'une agence nationale chargée de cette lutte pourrait voir le jour. La législation sera revue et son renforcement envisagé. Commentaire dans 24 Heures de Martial Saugy, directeur du Laboratoire d'analyse du dopage basé à Lausanne: «Avec les lois actuelles, il est par exemple très difficile d'inculper un médecin qui aurait donné des produits dopants à un sportif, simplement parce qu'il est protégé par le secret médical. C'est aberrant». Cette détermination du Gouvernement est une bonne nouvelle. On s'intéressera à la suite qui lui sera donnée.

\section{Löcher im Käse}

\section{Bernhard Gurtner}

Korrespondenz:

Dr. med. Bernhard Gurtner Eggstrasse 76

CH-8620 Wetzikon

gurtner.bernhard@bluewin.ch
Kürzlich hat eine Assistenzärztin berichtet, dass es an ihrer ökonomisch durchrationalisierten Weiterbildungsstelle unüblich, ja sogar unerwünscht sei, den Klinikleitern fachspezifische Fragen zu stellen. Mag sein, dass die gestressten Oberärzte und der medizinische CEO ihre Mitarbeitenden dazu erziehen wollen, Informationen selbst zu beschaffen, was heute dank raschem Zugriff auf Datenbanken leicht möglich ist. Vielleicht aber auch werden bohrende Fragen insgeheim gefürchtet, weil sie Wissenslücken in den führenden Köpfen aufdecken könnten. «Sie löchern mich», nennt man das in gepflegtem Hochdeutsch.

An unserem Regionalspital durften (oder mussten?) die Medizinstudenten im viermonatigen Praktikum an jedem Abendrapport «die Frage des Tages» stellen. Es war eine Offerte, ihnen Wissen und Erfahrungen ohne den Umweg über die Fachliteratur zu vermitteln. Die Frage des Tages sollte sie auch dazu ermuntern, alle Beobachtungen kritisch zu überdenken. Und schliesslich war der Informationsservice als kleine Kompensation gedacht für die oft unangenehme Rolle des Unterassistenten beim «bedside teaching». Dort wurde der «Uhu» als einziger befragt, wenn der Chefarzt annehmen musste, dass auch die Assistenzärzte oder der rückwärts beiständige Oberarzt die richtige Ant-

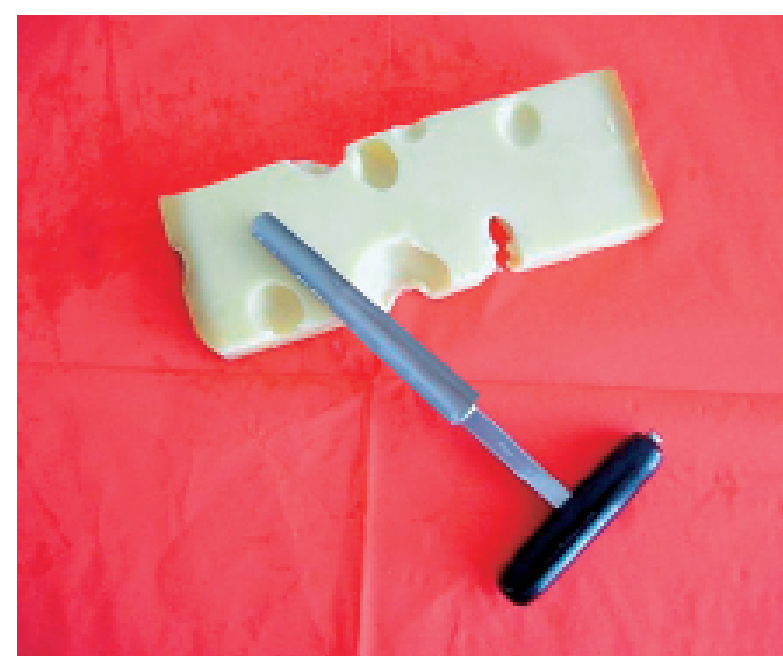


wort nicht gewusst hätten. Der Chef durfte ja die gelehrten Doktoren nicht vor den Patienten und dem Pflegepersonal blossstellen, während sich der junge Student noch im Gnadenstand der unverschuldeten Unwissenheit befand.

Erklärtes Nebenziel der Frage des Tages war es auch, Wissenshohlräume bei den Befragten anzustechen, wie die Löcher im Emmentaler Käse. Ein Praktikant bedankte sich denn auch mit dem originellen Geschenk eines scharf geschliffenen Käsebohrers. Das Fragen konnte bei den Kaderärzten einen Reifungsprozess auslösen, weil sie lernten, Nichtwissen unbefangen zuzugestehen. Im Wettlauf mit dem Studenten bemühten sie sich jeweils, bis spätestens am nächsten Morgen die gesuchte Antwort $\mathrm{zu}$ finden, was nicht immer gelang. (Weshalb ist der Herzspitzenstoss systolisch als Hebung zu spüren, obwohl sich doch das Herz in diesem Moment kontrahiert? Warum wird so oft empfohlen, Antibiotika 10 Tage lang zu verabreichen?)

Nun würde man meinen, die Studenten hätten die Frage des Tages geschätzt. Zu Beginn des Praktikums hatten aber viele erstaunliche Mühe, eine Frage anzubringen. Nicht, weil sie schon alles wussten oder sich zu wenig interessierten, sondern offensichtlich, weil sie befürchteten, sich durch Ignoranz zu blamieren. War das eine Spätfolge unseres Schulsystems?

Die vielen Fragen der die Welt entdeckenden und lustvoll begreifenden Kleinkinder finden die Eltern erst amüsant, dann allmählich lästig, wir Grossväter bewerten sie gerne als Zeichen einer wachen, zweifellos vererbten Intelligenz. Schon in der Primarschule wird aber leider allzuoft das Fragen ausgetrieben, die Fragenden werden $\mathrm{zu}$ Abgefragten umerzogen. Ihre Zeigefinger schiessen hoch, wenn sie die richtige Antwort zu wissen glauben. Nur die Mutigeren wagen es, aufzustrecken, weil sie etwas fragen möchten, und sie gelten bald als «dumme Frögli», die den
Ablauf des Unterrichts stören. Nichtwissen gibt schlechte Zeugnisnoten, es ist verdächtig auf Dummheit oder Faulheit oder beweist gleich beides.

Etwas nicht zu wissen, wird stets als peinlich empfunden, ein Gefühl, das wir bis ins Erwachsenenalter mitnehmen und das dazu verleitet, Wissenslücken möglichst zu verschleiern. So gab es auch unter unseren medizinischen Lehrmeistern nur wenige, die ganz offen dazu standen, eine Frage nicht beantworten zu können. Gar manche Klinikleiter oder Dozenten umgaben sich mit dem Nimbus der Allmacht und Allwissenheit, zumindest was ihr eigenes Fachgebiet betraf, Ausnahmen sind um so nachhaltiger in Erinnerung geblieben.

Seither ist das medizinische Wissen dermassen umfangreich und spezialisiert geworden, dass wir alle nur noch kleinste Fragmente überblicken und uns weitgehend auf den Neo-Cortex digital gespeicherter und abrufbarer Daten verlassen müssen. Zudem ist Wissen nicht mehr ein Privileg der (Ein-)Gebildeten, sondern allgemein zugänglich geworden, der ausgebildete Kranke hat die Vormacht des Arztes geschwächt.

Die Aus- und Weiterbildung darf sich daher nicht mehr darauf beschränken, Wissen mit kurzer Halbwertszeit einzutrichtern, das MultipleChoice-Artisten gewandt exmemorieren, am Krankenbett aber oft nicht von der Theorie in die Praxis umsetzen können. Klares Denken, Phantasie und Einfühlung sind gefragt. Wer an Rapporten zwei Minuten benötigt, um über etwas zu berichten, das sich in einem Satz zusammenfassen liesse, erweckt Zweifel an seiner Urteilsund Entschlusskraft in Notfallsituationen. Und wer ungeordnete ärztliche Berichte verfasst, darf sich nicht wundern, wenn er trotz Doktortitel nicht ernstgenommen wird. Einige Wissenslücken hingegen schaden nichts, sie lassen sich megagleitig stopfen. Doppelklick! 